\title{
RTFM-RT: a threaded runtime for RTFM-core - towards execution of IEC 61499
}

\author{
Andreas Lindner, Marcus Lindner, and Per Lindgren \\ Luleå University of Technology \\ \{andreas.lindner, marcus.lindner, per.lindgren\}eltu.se
}

\begin{abstract}
The IEC 61449 standard provides an outset for designing and deploying distributed control systems. Recently, a mapping from IEC 61499 to the RTFM-kernel API has been presented. This allows predictable real-time execution of IEC 61499 applications on light-weight single-core platforms. However, integrating the RTFM-kernel (bare-metal runtime) into potential deployments requires developing device drivers, protocol stacks, and the like. For this presentation, we apply the mapping from IEC 61499 to the RTFM-MoC task and resource model implemented by the RTFM-core language. The compilation from RTFM-core can be targeted to both, RTFM-kernel and the introduced runtime system RTFM-RT. In this paper, we detail the generic RTFM-RT runtime architecture, which allows RTFMcore programs to be executed on top of thread based environments. Furthermore, we discuss our implementation regarding scheduling specifics of Win32 threads (Windows) and Pthreads (Linux and Mac OS X). Using our RTFM-RT implementation for deployment, predictable IEC 61499 execution together with access to abovementioned operating system functions are achieved. For further developments, we discuss the needed scheduling options to achieve hard real-time and analysis required to eliminate deadlocks.
\end{abstract}

\section{INTRODUCTION}

Threads are the major means for modeling and implementing concurrency applications. The model of tasks and resources in contrast to threads is an alternative way that is slowly gaining acceptance. This can be seen in recent releases of task based libraries for concurrency in established development tools [1], [2].

IEC 61499 programs are compiled for threaded platforms, since available runtime systems are based on threads [3]. Threads are a programming model with a lot of complications and even arguable misconceptions [4]. On the other hand, thinking in terms of tasks and resources is both more natural and less complex. Our timed task and resource model RTFMMoC (outlined in Sections II-A and II-B) follows these terms to make reliable concurrent real-time systems available to a wider audience.

In this paper, we present RTFM-RT an abstract execution of RTFM-MoC under threaded environments and two implementations for the major operating systems. That way, programs of our task based model, written in RTFM-core [5], can be executed on mainstream and widespread thread based operating systems and libraries. RTFM-kernel [6] is another execution environment implementing the RTFM-MoC and aiming at highly efficient real-time execution on baremetal.

978-1-4673-7929-8/15/\$31.00 (c) 2015 IEEE
A mapping from IEC 61499 to RTFM-MoC and consecutively RTFM-RT allows more predictable and non-ambiguous execution, which in turn leads to more reliable applications. The proposal for the mapping of Function Blocks to the notions of tasks and resources in [7] gives an unambiguous execution semantics allowing to reason on properties of the system. These properties cover memory requirements as well as response times and overall schedulability.

The mapping extends the eventing mechanism and replaces the common event by two new event types. A synchronous event is a plain function call, while an asynchronous event marks the starting point of a chain of synchronous events associated with a priority. A chain of synchronous events is mapped to a task with deadline in RTFM-core. All stateful entities in an IEC 61499 model (i.e., data wires and Function Blocks) are mapped to resources protecting access to the state.

The proposed mapping has some limitations. A chain of synchronous events needs to be free of cycles, which is detectable and fixable. Furthermore, asynchronous events may not reoccur before their associated tasks are dispatched, which is ensured by timing analysis. With the outset from RTFM-core an additional extension to IEC 61499 would allow schedulability and response time analysis, i.e., adding timing properties and constraints to synchronous event chains [8]. Previous work relied on the SRP [9] based RTFM-kernel to ensure deadlock free execution. However, RTFM-RT currently does not guarantee deadlock freedom other than in special cases, thus deadlocks must be avoided by the mapping process.

\section{BACKGROUND}

RTFM-RT is part of a framework (RTFM-lang), which is intended to facilitate the development of reliable real-time applications. A major focus lies on providing a model for concurrent real-time programming, suitable for static analysis and run time verification.

To support a wide variety of users, especially less experienced in the field of real-time development, a domain specific programming language with an easy-to-use and readily understandable programming model is available. For this purpose, the RTFM-core language is based on the outset of concurrent tasks with timing constraints and named critical sections in contrast to commonly used threads. Figure 1 depicts the relationship between the components of the RTFM-lang framework, which are detailed in the following subsections. 


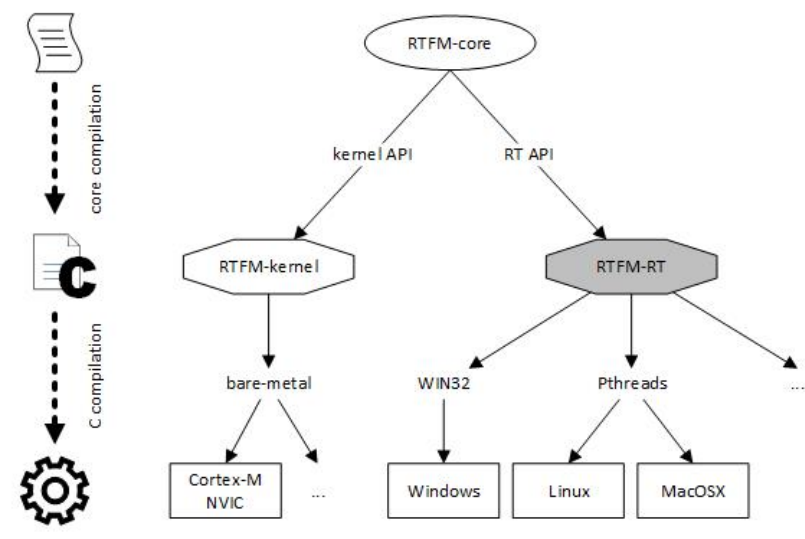

Figure 1. RTFM-RT as part of the RTFM-lang framework.

\section{A. RTFM-core Language}

RTFM-core is a programming language designed with concurrency support for the needs of lightweight embedded devices and particularly real-time systems with guaranteed response times, such as embedded sensors and actuators. Its model of computation (MoC), like for the whole RTFMlang framework, is reactive, based on tasks and (single-unit) resources combined with timing specifications. Tasks in contrast to threads are well-known to be suitable for real-time applications [10].

Tasks are the execution entities of a program, which encapsulate the executing code. Started by a triggering event, a task always runs to completion without waiting for additional events, i.e. a task has no option for blocking. Claiming a shared resource for access inherently involves synchronization, which is not considered to be blocking in RTFM-MoC. During execution a task may arbitrarily pend other tasks for asynchronous execution. Additionally, timing constraints (before and after) to pend a task's execution can be given.

Resources are logical entities to synchronize execution and protect critical sections within tasks, defined by a resource claim. Two or more tasks cannot claim the same resource at the same time, i.e., cannot enter their critical section. To enforce nested critical sections tasks can only be claimed in a LIFO (hierarchical) manner.

Figure 2 depicts the basic concepts of the RTFM-core language with its simplified grammar. Tasks (Task) and common functions (Func with parameters) are defined by a unique identifier ( Id) at top level, as well as the two special tasks Reset and Idle. Reset sets up the system, while Idle is an infinitely running task with lowest priority to support background activities (e.g., sleep mode). Identifiers have to be unique, which is ensured during the well-formedness check.

Functions are called with the sync keyword, while the asynchronous execution of a task is requested with either the pend or the async keyword. Pend and async optionally allow the specification of different timing constraints through before and after. Detailed information to the timing semantics is available in Section II-B.

Critical sections are delimited with a claim block within tasks and functions. The identifier of the claim denotes a

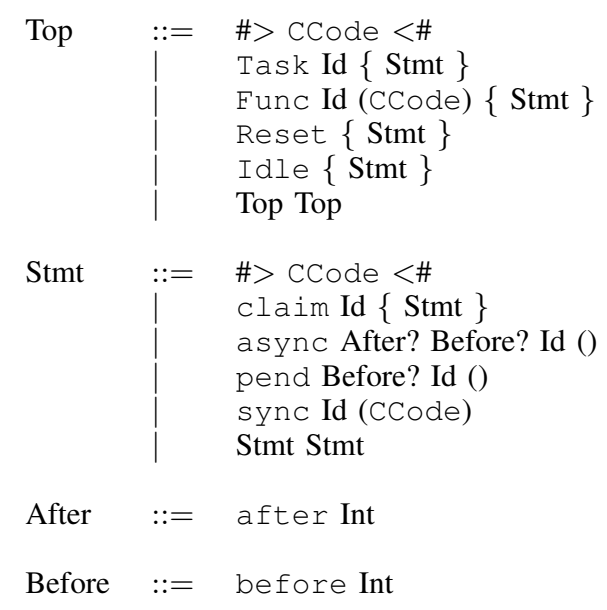

Figure 2. Simplified grammar for RTFM-core

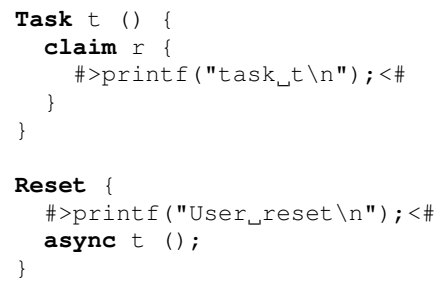

Listing 1. Example.core.

logical resource, which is claimed when entering the block and released when leaving the block. Multiple claim blocks that use the same identifier belong to the same resource and only one task can enter such a block at the same time.

This way each program specifies the task and resource structure of the application, as well as the timing dependencies between tasks. Actual program logic (commands, expressions, etc.) is embedded via $C$ code by usage of the escaping brackets $\#>$ and $<\#$.

Listing 1 illustrates a simple example program written in RTFM-core, which contains a task definition for Reset and a common task $t$. Reset is executed during the set up stage. It runs embedded $C$ code for a console output and requests the asynchronous execution of task $t$ with implicit timing constraints after 0 and before $\infty$. I.e., the execution is not delayed and there is no deadline specified. Task $t$ executes its embedded $C$ code within a critical section defined by claiming resource $r$.

This example does not show a particular functionality. It only exemplifies the main aspects of the RTFM-core language and is used later again in Section II-C for illustration of the compiler output.

\section{B. Timing Semantics}

Tasks of the RTFM-lang framework can only be executed within a defined time frame, i.e., Permissible Execution Window $(P E W)$. The borders of this time frame are always welldefined, either implicitly or explicitly, or a combination of both. The execution time of a task may never exceed the size of the $P E W$, but can be shorter. In the latter case, the execution starting point within the $P E W$ is left to the actual scheduling. 
Each task $t$ has two timing properties, a baseline $b l(t)$ and a deadline $d l(t)$. The baseline of each task is the earliest point in time, when the task execution is allowed. It specifies the lower bound of the $P E W$. The deadline defines the latest point in time, when the execution needs to be finished, which is the upper bound of the $P E W$.

Tasks can be triggered by another task (through pend or async) or by an external event. E.g., in case of the baremetal based RTFM-kernel an external event can be a hardware interrupt caused by a peripheral of a microcontroller.

Statement async:

The async statement in RTFM-core allows the declaration of two optional $P E W$ modifiers:

async (after Val)? (before Val)? Id()

When a task $t_{s}$ requests the execution of task $t$, then $t$ inherits the baseline of $t_{s}$, which can optionally be shifted by the value of the after modifier $($ after $(t))$ :

$$
b l(t)=b l\left(t_{s}\right)+a f t e r(t)
$$

If after is not specified then after $(t)=0$.

The value of the before modifier (before $(t)$ ) specifies the size of the $P E W$. The deadline of task $t$ is computed by its baseline and the $P E W$ size:

$$
d l(t)=b l(t)+\text { before }(t)
$$

For hard real-time systems, explicit deadlines should be specified, or inherited.

Statement pend:

The pend statement in RTFM-core allows only the declaration of the $P E W$ size by using the before modifier:

pend (before Val)? Id()

The baseline of a task $t$ is not inherited from the requesting task $t_{s}$ when using the pend statement. In that case the baseline of $t$ is set to the point in time when the pend statement is actually executed (time (pend $t)$ ):

$$
b l(t)=\operatorname{time}(\text { pend } t)
$$

The semantics of the before modifier follows exactly the one of the async statement and specifies the $P E W$ size.

\section{External event:}

An external event is mapped to the RTFM system using special tasks (not shown in the grammar in Section II-A). The baseline of such a task is implicitly the point in time when the causing event was captured (time $(e))$ :

$$
b l(t)=\operatorname{time}(e)
$$

The deadline is, similar to the pend and async statements, defined by a before modifier.

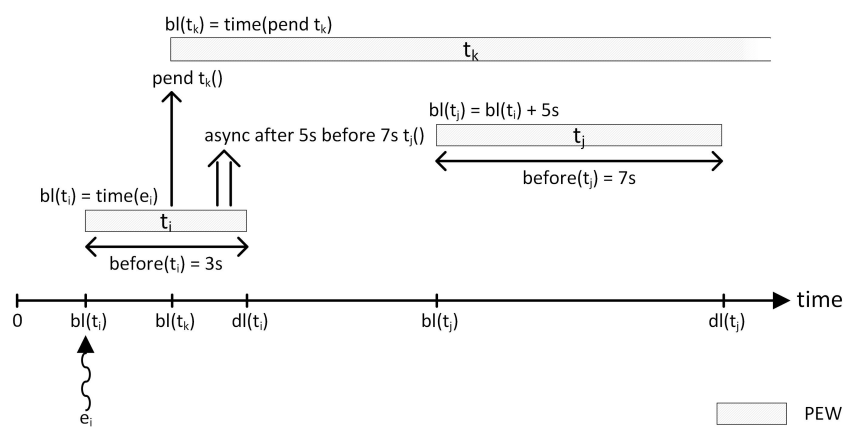

Figure 3. RTFM-lang timing example.

\section{Example:}

Figure 3 illustrates the timing diagram for a task $t_{i}$ that is triggered by an external event $e_{i}$ and two tasks $t_{j}$ and $t_{k}$, whose execution is requested by $t_{i}$. The baseline $b l\left(t_{i}\right)$ is computed by Equation 4 and $d l\left(t_{i}\right)$ set to $3 s$.

Execution of task $t_{j}$ is requested using the async statements. Both after and before modifiers are given. Baseline $b l\left(t_{j}\right)$ and deadline $d l\left(t_{j}\right)$ are computed using the Equations 1 and 2 respectively. Independently when async is executed within the $P E W$ of $t_{i}$, the $P E W$ of $t_{j}$ is always at the same position.

Task $t_{k}$ is requested by $t_{i}$ using the pend statement without specifying the before modifier. As a result $t_{k}$ has an infinite deadline. The baseline $b l\left(t_{k}\right)$, and thus the starting point of the $P E W$, is computed by Equation 3. This point in time is dependent on the actual execution of the pend statement, in contrast to the async statement for which the baseline is dependent on the senders baseline.

\section{Core Compiler}

Besides RTFM-RT as an execution environment for RTFMcore programs, the RTFM-lang framework provides also the RTFM-kernel execution environment (see framework overview in Figure 1). It executes RTFM-core programs on bare metal microcontrollers like the ARM Cortex-M3 series by exploiting the NVIC hardware for scheduling the tasks.

Both execution environments provide primitives as API for the task and resource based programming model in terms of $C$ macros and functions. These allow to initialize priorities for the tasks and resources, as well as pending tasks and locking or unlocking resources, which in turn affect the underlying run-time priorities. The APIs of RTFM-RT and RTFM-kernel slightly differ due to reasons of efficiency and implementation specific details.

Each RTFM-core program is compiled by the RTFM-core compiler to a $C$ program, which uses the specific API for the targeted execution environment. The following $C$ compiling stage invokes the respective set of $C$ macros and functions to produce the actual executable.

Task definitions in an RTFM-core program are represented as functions in the $C$ compilation, together with data structures storing their priorities and some other information. These functions are installed as interrupt service routines (ISRs) when 
executing on bare-metal hardware with the RTFM-kernel. The RTFM-MoC allows the same task $t$ to run more than once at the same time, i.e., task $t$ can be requested for execution multiple times with overlapping $P E W s$. Since an ISR can typically only be executing once at a time, it is necessary to create for each occurrence of a task execution request (pend or async) a task function (i.e., a task instance) in the $C$ code. Hereby, multiple ISRs are installed for the same task, which can run concurrently.

Task priorities are computed during the compilation based on the deadline specifications (before modifier). The highest priority is assigned to the task with the shortest deadline (deadline-monotonic priority assignment, [11]). Other approaches are possible.

All resources used in an RTFM-core program are represented as logical units with their names and ceiling values in the $C$ compilation. The ceiling of a resource is computed as the maximum of all task priorities that claim this resource. This allows for the application of the priority ceiling protocol during the execution under RTFM-kernel [9] and RTFM-RT [12] on some platforms.

Each async statement in an RTFM-core program specifies implicitly or explicitly (after modifier) the baseline for the new task. The RTFM-core compiler inserts code in the $C$ output to compute the starting point of the $P E W$ for the new task. The targeted runtime system aligns the scheduling for the task on its $P E W$.

Task and resource dependencies are analyzed during compilation by the RTFM-core compiler. Due to the SRP based scheduling under the RTFM-kernel, deadlocks can never occur. However, RTFM-RT has multi-core support and allows parallel execution of multiple tasks. In such case, the Coffman conditions for deadlock free execution are no longer guaranteed. To this end, each potential deadlock is detected at compile time and reported to the user, who can take countermeasures (e.g., by enforcing a global order of resource nesting). Passing off line analysis implies a guarantee for deadlock free execution, thus neither the run-time nor the application need to be designed for deadlock awareness. Future work will investigate alternative scheduling methods (e.g., MrsP [13]), which provide bounded blocking time.

Listing 2 is the simplified output of the RTFM-core compiler for the example program shown in listing 1. Resource $r$ and its ceiling value is stored in an enumeration for indexing and an array for the ceiling value (lines 1,2). Task indexes together with task priorities and function pointers are stored in the enumeration and arrays of lines $6-8$. The actual task instructions are embedded in plain $C$ functions (lines 10-22).

\section{Threads}

RTFM-RT executes RTFM-core programs on hosted environments like Windows and Linux. Concurrency support on such modern operating systems are provided by a thread architecture [14]. RTFM-lang tasks are mapped to threads and resources are modeled with mutexes. Semaphores are used to implement the request for asynchronous execution of a task, whereas a global clock is used to set its $P E W$ accordingly.

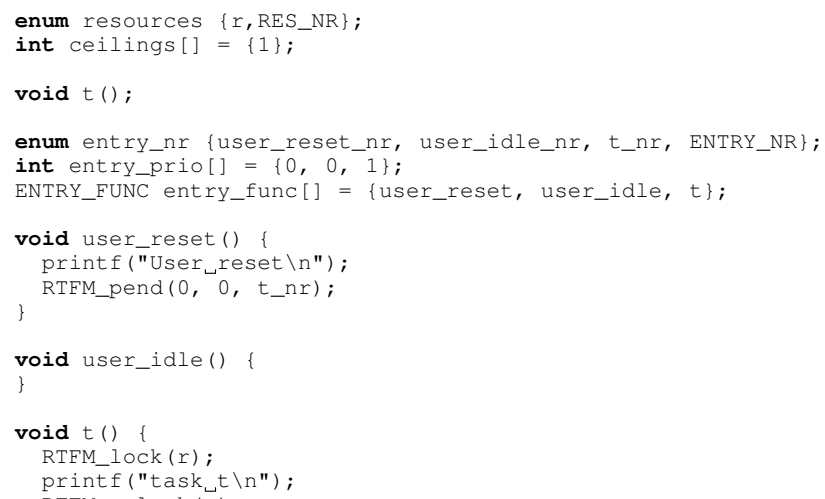

Listing 2. Example. c targeted to RTFM-RT (simplified).

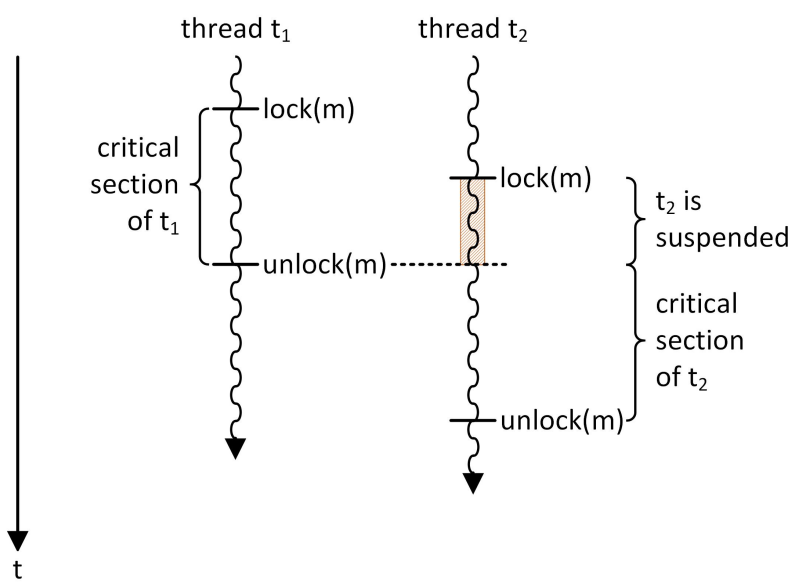

Figure 4. Mutex usage example.

\section{Mutex:}

In a threading environment a thread is an independent instruction sequence that can be executed in parallel to other threads. A critical section of a thread is a block of instructions, which potentially interfere with instructions from other threads (e.g., access to the same memory location). To prevent errors in the program execution, these blocks may not be executed concurrently.

A mutual exclusion (mutex) object is a data structure with two atomically executed access operations, lock and unlock. A thread that owns a mutex (i.e., executed lock) is the only one who can release (i.e., execute unlock) it again and no other thread can lock it in the meantime. By delimiting all critical sections in concurrent threads with lock and unlock instructions on the same mutex, none of these sections are executed at the same time.

Figure 4 illustrates the functionality of a mutex $m$. Threads $t_{1}$ and $t_{2}$ run in parallel and have each a critical section. The first thread that reaches its critical section $\left(t_{1}\right)$ can take the ownership of the mutex by executing lock (m)). As soon as the second thread $\left(t_{2}\right)$ reaches its critical section, its execution is suspended until it can take the ownership of the mutex (i.e., unlock (m)) by $t_{1}$ ). 


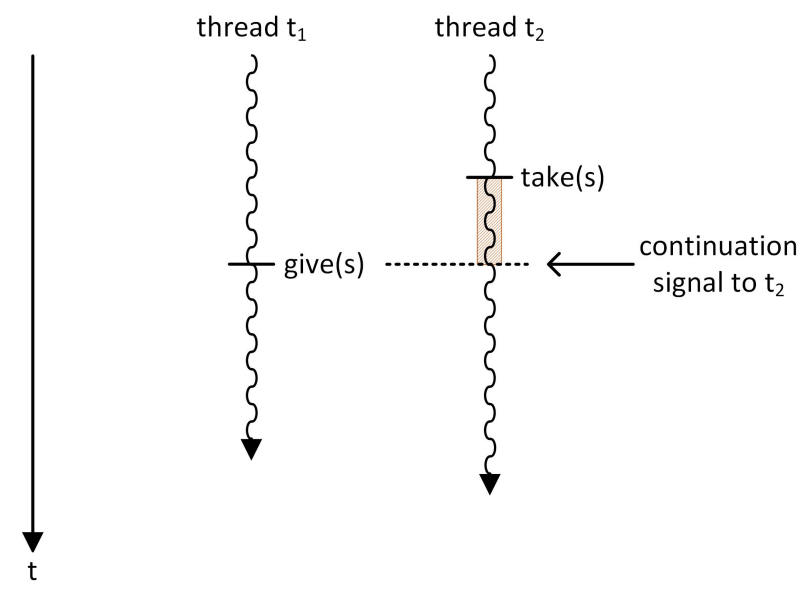

Figure 5. Semaphore usage example. The semaphore $s$ is initialized to 0 .

\section{Semaphore:}

Semaphores are intended for another type of synchronization in between threads. Their main purpose is for access control and signaling in concurrent scenarios. Similar to mutexes there are atomically executable lock (take) and unlock (give) operations available for semaphores. But in contrast to mutexes there is no ownership for a semaphore. Each thread with access to the semaphore can release it, if it is already locked.

A special type of semaphores, used by the RTFM-RT, are binary semaphores. General semaphores specify the maximum number of possible take operations. Each take operation decreases a counter down to 0 , while each give operation increases it up to the maximum. Binary semaphores set the maximum to 1 , i.e., take and give operations alternate.

Figure 5 shows a simple example of using a semaphore $s$. Thread $t_{2}$ executes its instructions until it tries to take the semaphore (take $(s)$ ). Since the semaphore was not yet given (give $(s)), t_{2}$ has to wait for the semaphore. As soon as $s$ is given by $t_{1}$ (give $(\mathrm{s})$ ), $t_{2}$ can continue. That way $t_{1}$ can signal $t_{2}$ for continuation, e.g., after $t_{1}$ prepared work for $t_{2}$.

\section{GENERIC IMPLEMENTATION OF RTFM-RT}

The main focus of the RTFM-lang framework lies on baremetal execution with the RTFM-kernel scheduling system. However, RTFM-RT (as part of RTFM-lang) aims at executing RTFM-core programs on feature-rich operating systems, like Windows and Linux. That gives a broader range of target platforms for running RTFM-MoC based programs, as well as access to built-in functions like protocols and drivers.

RTFM-MoC uses tasks and resources for concurrency, which is a significant difference to the thread models used by common operating systems [10]. RTFM-RT defines a mapping from the RTFM-MoC to a generic thread model, which is the basis for the RTFM-RT architecture. Specialized implementations for the major operating systems and their used thread models (Win32 threads [15], POSIX threads [16]) are available.

During run time, RTFM-RT verifies the specified timing requirements by checking all deadlines. After termination of each task, its deadline (derived from the program specification) is compared to the global time value.

\section{A. Requirements}

RTFM-RT is designed as a runtime system for RTFM-lang, i.e., it has to satisfy the RTFM-MoC. The three main aspects of the RTFM-MoC are tasks, resources, and timing properties given for the tasks. The API of RTFM-RT provides functionality to execute RTFM-core programs while preserving RTFMMoC semantics during execution (i.e., scheduling). Therefore, tasks and resources are defined together with their priorities and ceilings, given as output from the RTFM-core compiler. Instructions for requesting asynchronous task executions are available, which keep all $P E W$ timing properties for computing the baselines and checking the deadlines during run time.

Table I shows implementation specific differences of the RTFM-kernel and the RTFM-RT with respect to the RTFMMoC. The RTFM-kernel exploits directly the peripheral hardware (ISRs, interrupt masking, timers), which makes it very efficient. Without direct access to the hardware RTFM-RT uses instead available threading architectures for scheduling and representing tasks, resources, or baselines (threads, mutexes, OS clocks).

\begin{tabular}{|l||c|c|}
\hline \multicolumn{1}{|c||}{} & RTFM-kernel & RTFM-RT \\
\hline \hline Parallel execution & Single-core only & Multi-core possible \\
\hline Representation of tasks & ISRs & Threads \\
\hline Representation of claims & Interrupt masking & Mutexes \\
\hline Task scheduling & SRP & $\begin{array}{c}\text { POSIX: FIFO } \\
\text { Windows: Win32 }\end{array}$ \\
\hline Resource locking & Priority ceiling & $\begin{array}{c}\text { POSIX: priority ceiling } \\
\text { Windows: priority classes }\end{array}$ \\
\hline Deadlocking & Deadlock free & Optional deadlock detection \\
\hline Timing & Hardware timers & OS clocks \\
\hline
\end{tabular}

Table I. COMPARISON OF RTFM-KERNEL AND RTFM-RT.

\section{B. Static Design}

RTFM-RT executes arbitrary programs that follow its API. A program for RTFM-RT is a $C$ code file with certain data structures and function definitions (see Listing 2). Different variables store the task and resource properties (i.e., priorities and ceilings), while the actual task instructions are encapsulated in functions.

These data structures and function definitions form the configuration (as an application file) for the runtime system. It is used for static initialization of threads (one per task), mutexes (one per resource), and semaphores (one per task for signaling). Task code (encapsulated in functions) can use the API instructions pend and async for task execution requests, as well as the API instructions lock and unlock for claiming and releasing resources. Timing properties for each task are encoded in the API instructions pend or async that request the execution of the task.

The RTFM-core compiler transforms RTFM-core programs into the application file ( $C$ code), which follows the described API specification. Subsequently, a $C$ compiler takes this application file together with the RTFM-RT base system (available as $C$ code) and outputs an executable for the target platform. 


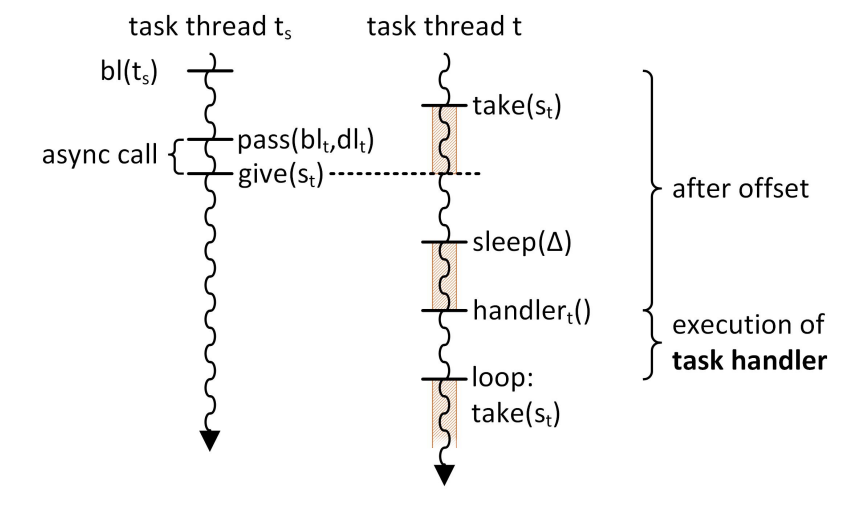

Figure 6. Pending a task thread in RTFM-RT.

\section{Dynamic Design}

Based on the application file discussed in the previous section, the RTFM-RT system is set up and can be started. The main thread executes the following steps:

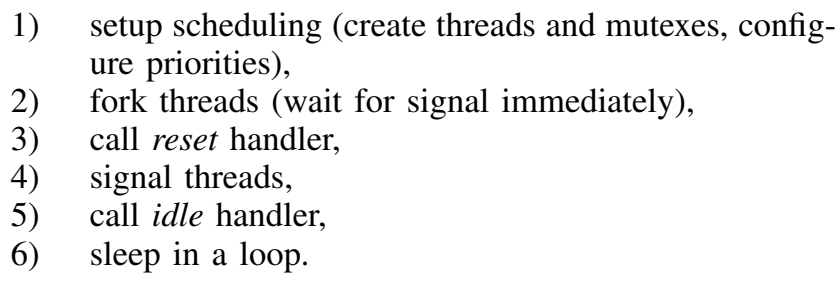

Each task instance is represented by one thread, which executes the following steps:
1) wait for async semaphore,
2) sleep $\Delta$ (if baseline in future),
3) call task handler,
4) jump to 1).

Step 2 is the offset for the new baseline of the task, i.e., the starting point of the $P E W$. It is computed using the after modifier in the RTFM-core program. The actual task execution (step 3) may not begin outside of the PEW.

Figure 6 illustrates a request from source task $t_{s}$ for asynchronous execution of task $t$. Task $t_{s}$ starts its execution after the defined baseline $b l\left(t_{s}\right)$, while task $t$ is in wait mode for its semaphore take $\left(s_{t}\right)$. An async call for task $t$ is composed of passing data over to the target thread (i.e., the baseline and deadline for $t$ ) and signaling the target thread (give $\left(s_{t}\right)$ ). This wakes up the thread of task $t$ to handle the receiving data and fall asleep again for time period $\Delta$, if the baseline lies in the future. The actual task execution takes place after entering the $P E W$.

Figure 7 depicts the interaction of the main and task thread from the RTFM-core example 1. It shows also the usage of a mutex (lock $\left(m_{r}\right)$ and unlock $\left.\left(m_{r}\right)\right)$ to delimit a critical section. In case of another task thread using the same mutex (i.e., claiming the same resource), a mutual exclusion of both critical sections would be ensured by the thread system.

\section{SPECIFIC IMPLEMENTATIONS}

Windows and Linux/Mac OS X are using different threading architectures. While Windows has its own implementation

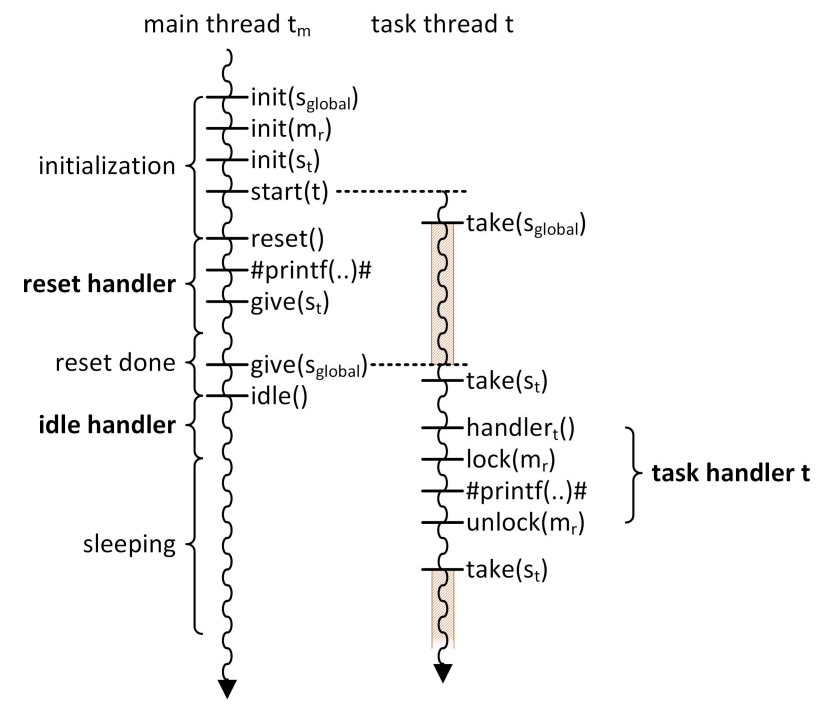

Figure 7. Main thread and task thread of example Listing 1 running under RTFM-RT.

with Win32 threads [15], Linux and Mac OS X are using the standard POSIX threads [16]. The different architectures support different mechanisms, which treat priorities and priority inversion in different ways. A separate runtime system for each target platform considers the differences.

\section{A. PTCORE}

PTCORE is the RTFM-RT version for POSIX threads (Pthreads) supported by Linux and Mac OS X. Pthreads support various thread scheduling policies. We opted for the SCHED_FIFO scheduling, which is the one with minimal overhead and closest to the behavior of the hardware based RTFM-kernel scheduling regarding predictability.

SCHED_FIFO scheduling defines an implementation dependent number (between 32 and 99) of available priority levels. There is no time slicing for running threads. Once a thread is started, it runs until:

- a new thread with higher priority is ready (preemption),

- it is blocked blocked by requesting an I/O operation,

- it is trying to lock an already locked mutex,

- or it yields.

Threads that are ready for execution are kept in a FIFO queue, whereas each priority has its own queue. Preempted threads are inserted at the beginning, while new and yielding threads are inserted at the end. The scheduling selects for next execution the thread with the highest priority that is currently unscheduled and ready.

RTFM-RT uses for Pthread execution the PTHREAD_PRIO_PROTECT mutex protocol. A thread that is requesting a mutex (lock) gets it and continues execution if the mutex was not locked. The priority of the thread changes to the maximum of the current priority and the priority ceiling of the mutex. The claiming thread is blocked, if the mutex was already locked, until the mutex is unlocked and the scheduling rules allow to continue the blocked thread. 
When a thread releases a locked mutex the priority of the thread is reverted to the old one valid before locking the mutex. All other threads waiting for this mutex can be scheduled for execution based on the applicable policy.

\section{B. WINCORE}

WINCORE is the RTFM-RT version for Win32 threads provided by Windows. In contrast to Pthreads the scheduling policy is predefined and not adjustable. The applied policy is similar to the Pthread SCHED_RR policy, but with random boosting to solve starving priority inversion.

Within a process there are seven settable priority levels available for the threads. Priorities of threads that are ready for execution are randomly increased by a priority boosting strategy. I.e., the dynamic priority of a thread is its defined base priority plus a boost value, which is set to a random value at a random time.

From the set of threads that are ready for execution, the one with the highest dynamic priority is selected for scheduling. The execution is regularly interrupted to share the processing time in between the threads (i.e., time slicing). The next thread with the highest priority is selected in a round robin pattern.

The mutex protocol in Windows has no concept of priority ceiling. Locking and unlocking of a mutex only manages the blocking of threads in order to enforce mutual exclusion. The random priority boosting strategy solves the thread starvation due to priority inversion.

A thread, which is currently executing, can lock a mutex and immediately continue its execution, if the mutex was not locked. The thread is blocked and cannot be scheduled again, if the mutex was locked. In this case, the execution cannot continue before the mutex is unlocked and the scheduler selects this thread again for execution based on the scheduling policy.

\section{RELATED WORK}

RTFM-core is developed as an intermediate language for the object oriented application level language RTFM-cOOre [17]. Modeling IEC 61499 in RTFM-cOOre is feasible (e.g., stateful entities as objects), but a different code emission for Function Block algorithms is required, since embedded $C$ code is not possible.

Erlang is a functional programing language with concurrency and distribution support in its runtime system [18]. Message passing is the applied technique for data communication between processes, which makes synchronization primitives obsolete. However, Erlang has no means to specify timing properties and was developed for soft real-time. Therefore, our RTFM-core approach gives this benefit to IEC 61499 execution.

Due to its easier comprehension, the integration of taskbased programing models become popular in modern languages or libraries (e.g., Java [2], C\# [1]). Like Erlang, they have no compile-time timing analysis and are also not intended for hard real-time applications.

Other non-mainstream approaches include synchronous languages (e.g., Signal [19], Lustre [20], Esterel [21]) and task-based languages like Ada [22]/SPARK Ada [23]. The effort needed to introduce them in development is in general considered to be high, since their programming paradigms largely differ from industrial state-of practice. A common fear is the implication of large costs for migrating legacy solutions and the revision of the whole design process in order to fully exploit the potential.

RTFM-lang on the other hand is very suitable for executing IEC 61499 models. It has the goal of facilitating embedded development and concurrent programming in general by giving guarantees for execution and compile-time timing analysis. The work in [8] proposes a real-time semantics to the IEC 61499 standard based on the RTFM-MoC. It details the mapping of timing requirements from IEC 61499 programs to the timed task and resource model of RTFM-core. In prior work, the RTFM-kernel for efficient deployment onto singlecore platforms was developed.

In [24] various development tools and run-times for IEC 61499 are compared against each other in terms of compatibility. Looking at FBDK/FBRT, the order of execution within an FB network is similar to the resulting execution of our FB mapping. Events are modeled as synchronous function calls and they are handled immediately. All run-times for ISaGRAF, FBDK, and 4DIAC (i.e., ISaGRAF Runtime, FBRT, and FORTE) are generic and provide an abstract interface for the applications. In contrast to this, our run-time is compiled together with its application allowing more narrow analysis of constraints at a lower level. Furthermore, this ensures a higher degree of specialization and efficiency.

\section{Future EXTENSIONS TO HARD REAL-Time}

RTFM-RT is dependent on the underlying thread architecture. Neither Windows (WINCORE) nor Linux (PTCORE) provide a thread scheduling with guaranteed time boundaries, which makes hard real-time execution of RTFM-core impossible. Xenomai is a Linux kernel extension, where the Linux system steps back and appears as a background task behind Xenomai tasks [25], which are hard real-time tasks. It is hybrid solution giving both advantages, real-time capabilities together with legacy Linux support.

WinCE is another platform with hard real-time support [26] and high level options, like protocol and driver interfaces. It is by design suited to industrial applications in contrast to Win32 and thus another option for further investigation for the RTFMRT system.

In order to achieve deadlock free execution static analysis is performed at compile time, reporting programs with potential deadlocks. The designer can then revise the program to obtain a system free of deadlocks. Other automatic methods under investigation are bound blocking time multi-core scheduling (MrsP) and lock transformations in the program. The execution of IEC 61499 programs is free of deadlocks as long as events are treated asynchronously. By transforming asynchronous events to synchronous ones where possible, threading overhead can be reduced. However, we need to ensure that synchronous chains will not appear and potential deadlocks are not introduced by the optimization. The techniques and optimization tradeoffs are targeted for future work.

Another extension to make the execution safer can be memory protection specifically for shared resource memory. 
Therefor reconfiguration of memory access hardware (MMU or MPU) is applied when entering or leaving claim blocks. The MMU supports distinction of virtual memory configurations for each schedulable entity (i.e., task). The MPU allows definition of a limited number of access regions, what can be further optimized with an intelligent memory layout.

\section{CONCLUSION}

The industry standard IEC 61499 lacks a clear specification for predictable execution. In the presented paper, we use a defined execution and timing semantics (RTFM-MoC) as an outset for a runtime system (RTFM-RT). The proposed RTFM4-FUN mapping [7] from IEC 61499 models to RTFM-MoC is used for the execution under RTFM-RT.

We developed the generic runtime RTFM-RT to execute RTFM-core programs on threaded platforms. It is intended for all threaded environments, which makes it hardware independent and concrete implementations exist already for Windows and Linux/Mac OS X. RTFM-RT can also handle multiple execution units (i.e., multi-core platforms) in opposition to the RTFM-kernel.

Both runtime implementations suffer hard real-time support (due to the used thread systems) and potential deadlock occurrence. Among others, that leaves room for improvement. Memory protection support to isolate shared memory access can be added to further improve safety in terms of reliability.

PTCORE, as one possible implementation, is closer to interrupt based scheduling (e.g., RTFM-kernel or SLOTH [27]) and hence more predictable. WINCORE on the other side is closer to thread abstraction, where priorities are only provided to allow for pushing best effort execution in a direction (i.e., favor processing of one thread instead of another).

Next steps will be further investigation on support of hard real-time for RTFM-RT (see Section VI), as well as adding support for timing specifications to the IEC 61499 standard. This allows execution of IEC 61499 programs with specified timing behavior, while still providing access to high level operating system functionality (i.e., legacy support and compatibility).

\section{REFERENCES}

[1] C. Nagel, B. Evjen, J. Glynn, K. Watson, and M. Skinner, Professional C\# 2012 and .NET 4.5, 1st ed. Birmingham, UK, UK: Wrox Press Ltd., 2012.

[2] T. Peierls, B. Goetz, J. Bloch, J. Bowbeer, D. Lea, and D. Holmes, Java Concurrency in Practice. Addison-Wesley Professional, 2005.

[3] A. Zoitl, Real-Time Execution for IEC 61499. Durham, North Carolina: International Society of Automation, 2008.

[4] E. A. Lee, "The problem with threads," Computer vol. 39, no. 5, pp. 33-42, May 2006. [Online]. Available: http://dx.doi.org/10.1109/MC.2006.180

[5] P. Lindgren, M. Lindner, E. Fresk, D. Pereira, and L. Pinho, "Rtfm-core Language and implementation," 2014, paper presented at Embedded Systems Week, New Delhi, India.

[6] J. Eriksson, F. Haggstrom, S. Aittamaa, A. Kruglyak, and P. Lindgren, "Real-time for the masses, step 1: Programming API and static priority SRP kernel primitives." in SIES. IEEE, 2013, pp. 110-113. [Online] Available: http://dblp.uni-trier.de/db/conf/sies/sies2013.html

[7] P. Lindgren, M. Lindner, A. Lindner, J. Eriksson, and V. Vyatkin, "RTFM-4-FUN," in SIES. IEEE, 2014.
[8] P. Lindgren, M. Lindner, A. Lindner, V. Vyatkin, D. Pereira, and L. Pinho, "A Real-Time Semantics for the IEC 61499 standard."

[9] T. Baker, "A stack-based resource allocation policy for realtime processes," in Real-Time Systems Symposium, 1990. Proceedings., 11th, Dec. 1990 , pp. $191-200$.

[10] N. Wirth, "Tasks versus threads: An alternative multiprocessing paradigm." Software - Concepts and Tools, vol. 17, no. 1, pp. 6-12, 1996

[11] N. C. Audsley, A. Burns, M. Richardson, and A. Wellings, Deadline monotonic scheduling. Citeseer, 1990

[12] L. Sha, R. Rajkumar, and J. Lehoczky, "Priority inheritance protocols: an approach to real-time synchronization," Computers, IEEE Transactions on, vol. 39, no. 9, pp. 1175-1185, Sep 1990.

[13] A. Burns and A. Wellings, "A schedulability compatible multiprocessor resource sharing protocol - mrsp," in Real-Time Systems (ECRTS), 2013 25th Euromicro Conference on, July 2013, pp. 282-291.

[14] G. Taubenfeld, Synchronization Algorithms and Concurrent Programming. Upper Saddle River, NJ, USA: Prentice-Hall, Inc., 2006.

[15] A. Cohen and M. Woodring, Win32 multithreaded programming building thread-safe applications. O'Reilly, 1997.

[16] S. Kleiman, D. Shah, and B. Smaalders, Programming with threads. Mountain View, Calif. Sun Soft Press Upper Saddle River, NJ: Prentice Hall, 1996, lccopycat. [Online]. Available: http://opac.inria.fr/record=b1091557

[17] P. Lindgren and M. Lindner, "Rtfm-core: Course in compiler construction," 2014, paper presented at Embedded Systems Week, New Delhi, India.

[18] J. Armstrong, Programming Erlang: Software for a Concurrent World. Pragmatic Bookshelf, 2007.

[19] P. LeGuernic, T. Gautier, M. Le Borgne, and C. Le Maire, "Programming real-time applications with signal," Proceedings of the IEEE, vol. 79, no. 9, pp. 1321-1336, Sep 1991.

[20] D. Pilaud, N. Halbwachs, and J. Plaice, "Lustre: A declarative language for programming synchronous systems," in Proceedings of the 14th Annual ACM Symposium on Principles of Programming Languages (14th POPL 1987). ACM, New York, NY, vol. 178, 1987, p. 188.

[21] G. Berry, P. Couronne, and G. Gonthier, "Synchronous programming of reactive systems: an introduction to esterel," 1987.

[22] D. Mandrioli, R. Zicari, C. Ghezzi, and F. Tisato, "Modeling the ada task system by petri nets," Computer Languages, vol. 10, no. 1, pp. 43 - 61, 1985. [Online]. Available: http://www.sciencedirect.com/science/article/pii/0096055185900104

[23] J. Jedryszek and A. Pinninti, "Spark ada programming for arm-based device (beagleboard-xm)." [Online]. Available: http://jj09.net/wp-content/uploads/2014/06/SPARKAda-programming-for-ARM-based-deviceBeagleBoard-xM.pdf

[24] J. H. Christensen, T. Strasser, A. Valentini, V. Vyatkin, A. Zoitl, J. Chouinard, H. Mayer, and A. Kopitar, "The IEC 61499 Function Block Standard: Software Tools and Runtime Platforms," ISA Automation Week, 2012

[25] A. Barbalace, A. Luchetta, G. Manduchi, M. Moro, A. Soppelsa, and C. Taliercio, "Performance comparison of vxworks, linux, rtai and xenomai in a hard real-time application," in Real-Time Conference, 2007 15th IEEE-NPSS, April 2007, pp. 1-5.

[26] S. Phung, D. Jones, and T. Joubert, Professional Windows Embedded Compact 7. Wrox, 2011.

[27] W. Hofer, D. Lohmann, F. Scheler, and W. Schroder-Preikschat, "SLOTH: Threads as Interrupts," in Proceedings of the 30th IEEE RealTime Systems Symposium (RTSS '09), pages 215228, IEEE Computer Society, 2009, ISBN 978-0-7695-3875-4, 2009. 